\title{
Emotion, Discretionary Behavior, and IT Effectiveness
}

\author{
${ }^{1}$ Manhui Huang ${ }^{*},{ }^{2}$ Jinghua Xiao and ${ }^{3}$ Kang Xie \\ ${ }^{1}$ Information Science School, \\ Guangdong University of Finance and Economics, \\ Guangzhou 510320, China \\ ${ }^{2,3}$ Business School, \\ Sun Yat-sen University, Guangzhou 510275, China \\ ${ }^{1}$ huangmanhui@126.com, ${ }^{2}$ Insxjh@mail.sysu.edu.cn, ${ }^{3}$ mnsxk@mail.sysu.edu.cn
}

In organizations with broadly defined and nebulous job description, the employees need to take initiative in accomplishing tasks. Discretionary behaviors may contribute more than inrole behaviors to performance in this situation Researchin organizational behavior has shown that emotion plays a critical role in predicting diseretionary behavior. However, traditional measurements of information technolog. (IT) use can not capture all the discretionary behaviors and identify effective IT isage, and most IT use research focuses on the cognitive antecedents, while pay little attentron to its emotion antecedents. Therefore, it is important to explore the emotion antecedents of IT effectiveness. Based on the results of previous discretionary behavior research we make a preliminary recognition of discretionary behaviors in $T$ context and propose a conceptual model, which shows the important role of emotion for IT effectiveness, and the relationships among emotions, discretionary IT behaviors, and IT effectiveness. The conceptual model proposed in this paper not only provides an explanation of how emotion affects IT effectiveness and why simply IT use may not always lead to IT effectiveness, but also reveals the areas which further research is needed to better understand T use and IT effectiveness.

Keywords: IT effectiveness; discretionary behavior; IT use; emotion

\section{Introduction}

Successtul application of information technology (IT) is beneficial to individual performance and organizational performance. To achieve IT impact, one prerequisite is individual IT use $[1,2]$. This has driven a lot of research focused on the antecedents of IT use $[3,4]$. However, IT usage may not necessarily lead to improved individual performance because inappropriate IT use may have no benefits, and discretionary behaviors in IT usage will determine the impact of IT. Previous research in organizational behavior has shown that emotion is an important predictor of discretionary behavior. However, discretionary behavior remains an under-studied topic in IT field. In this study, we focus on IT effectiveness which refers to the impact of IT on individual job performance, and try to recognize the discretionary behaviors and analyze their emotion antecedents and their contributions to IT effectiveness.

${ }^{*}$ Corresponding author 
There are several reasons for the importance of recognizing discretionary behaviors in IT context and analyzing the relationship between discretionary behaviors and IT effectiveness. First, IT use does not necessarily result in IT effectiveness, as improper IT usage may have no contribution to job performance [1]. Second, most extant research used frequency of use, time of use or number of access to measure IT use behavior[5]. However, these variables may not represent effective IT use. Third, research in organizational behavior has shown that individual job behaviors can be divided into in-role behaviors and discretionary behaviors. In jobs requiring individual to take initiative, discretionary behaviors contribute more than inrole behaviors to the overall job performance[6,7]. In-role behavior is related directly to job description, and discretionary behavior refers to the voluntary or unexpected actions beyond job description[8]. Similarly, we believe IT usage behaviors can be divided into in-role behaviors and discretionary behaviors, and discretionary behaviors may play an important role for IT effectiveness. Fourth, in organizations with wide application of T, Job description is broadly defined and nebulous. This requires employees to take initiative in-accomplishing tasks. With less in-role behaviors, discretionary behaviors become more important for achieving job performance in such organizations [7]. Additionally, the application of IT is a teamwork [9], which means that it is important to interact with other people to achieve IT effectiveness, and more interactions with other people will lead to greater opportunities to display discretionary behaviors [7]. Therefore it is necessary to identify the discretionary behaviors in IT use and make further analys sis about their antecedents and their outcome.

Another limitation in extant IT use literature is that most research about the antecedents of IT use bases primarily on cognitive models such as the technology acceptance model [2, 10], theory of reasoned action [11], theory of planned behavior [12], and the unified theory of acceptance and use of technoregy [4]. This line of research has contributed to our understanding that perceptions and beliefs about IT such as perceived usefulness, perceived ease of use, perceived compatibility, perceived behavioral control, and subject norm are important to predict IT use. However, research in organizational behavior and human resource management has shown that the antecedents of behavior are complex and multifaceted, andemotion may also play an important role in predicting individual behavior especially the discyetionary behavior $[7,13,14]$. Therefore, it is important to analyze the emotion antecedents of discretionary IT usage behavior and their relationship with IT effectiveness.

The purpose of this paper is to (1) preliminarily recognize the discretionary behaviors in IT use, (2) show how emotions affect IT effectiveness through discretionary behaviors, and (3) explain whysome individuals are willing to take initiative to make IT have positive impact on their work as well as organizational performance, while some individuals are trying to resist the application of IT.

The neminder of this paper will first review the research on emotions, job performance and IT effectiveness. A new framework that presents the important role of emotions for IT effectiveness is then developed. Finally, contributions of this study and suggestions for future research are discussed.

\section{Emotions and IT effectiveness}

\subsection{Emotions and IT use}

Emotion is a functional mechanism or mental state when highly organized, ongoing activity is interrupted to force attention on urgent events that are relevant to physiological needs or that induce disturbing cognitive associations, such as threats to esteem $[3,15]$. That is, emotion is an adaptive function or mental state in response to environmental events [3, 15]. 
Emotion energizes individual physiologically and then influences individual behavior $[3,15]$. Emotion has specific referents and it arises in response to the appraisal of an event which is perceived as relevant and important to the individual [3]. Generally, emotions can be divided into two categories including negative emotions and positive emotions. Negative emotions include anger, anxiety, boredom, and depression, whereas positive emotions include cheerfulness, contentment, enthusiasm, and happiness [15]. The application of IT will interrupt the sequence of events in individual user routine, and will trigger his/her emotion [3].

Past IT research has examined the effects of some emotions on attitude, beliefs, and IT use. Generally, the emotions generated in two different periods were studied. One is the emotions generated in the anticipation period $[3,16]$, the other is the emotions generated in impact period $[3,17,18]$. Results show that specific emotion (e.g., anger, anxiety, exclitement, and happiness) generated in both the anticipation period [3] and the impact period [18, 19] are related to IT use.

However, the effects of emotions on IT effectiveness are left largely umexplored. Most extant research has focused on the emotions generated in the impact period [3], while the research on the emotions generated in anticipation period and implementation period is still limited. Yet research in organizational behavior has shown that emotions have long-lasting effects on behavior $[15,20]$. Additionally, as discussed above, iT use does not necessarily lead to IT effectiveness. Therefore, research about the effects of emotions on IT effectiveness is needed.

\subsection{Discretionary behaviors and Ir elfectiveness}

Research in organizational behavior and human resource management has shown that individual job performance is decided by two kinds of behaviors: in-role behaviors and discretionary behaviors. The latter includes organizational citizenship behaviors (OCB) and counterproductive work behaviors (CWB). Organizational citizenship behaviors are prosocial behaviors exceeding the normative expectations of the organization [7]. Examples of OCB include helping coworkers and attending functions that are not required [14]. Counterproductive work behayiors are intention behaviors that go against the legitimate interests of the organization [7]. Examples of CWB include aggression or sabotage [15]. Discretionary behaviorplays an important role in job performance, as past research has shown that OCB and job performance have moderate to strong positive relationship, and CWB and job performance have profound negative relationship [7].

Similarly, in IT context, we believe IT use should include in-role behavior and discretionar behavior. First, although management may explicitly require employees to use IT, it is diffycult to specify the details concerning its actual application. Second, how to use IT may invorve many discretionary behaviors because the employees may decide whether or not to take, initiative to use IT effectively. Therefore, it is necessary to recognize the discretionary behaviors in IT use. Here we divide IT use behavior into in-role IT use behavior and discretionary IT use behavior. In-role IT use behavior refers to fulfilling the management requirements to learn and use IT such as (a) participating the training activities organized by the company according to the requirements, (b) inputting data to IT systems according to the requirements, and (c) outputting data from IT systems according to the requirements.

Discretionary IT use behavior can be divided into OCB and CWB. The OCB in IT use means taking initiative beyond the requirements of management to make sure that IT can help improve not only individual but also organizational performance. As application of IT is a teamwork, and needs cooperation among different users or between IT user and IT personnel [9], beyond the in-role IT use, there are some other behaviors which will contribute to IT 
effectiveness and be difficult to be prescribed by management. We refer the set of OCB in IT use as discretionary IT cooperation behavior.

Based on reviewing the discretionary behavior literatures and our pilot interview with the Chief Information Officers (CIO) in 3 companies, a set of discretionary IT cooperation behaviors are generated as below: (a) taking initiative to learn the skill about IT use to make sure they can operate IT well; (b) reporting the operation problems they met immediately to make sure IT work well; (c) providing suggestions to make data input and output more conveniently and more friendly; (d) eagerly helping other people to solve IT problems, as helping is critical to conduct cooperation behavior [21]; (e) taking initiative to discuss with colleagues about IT solutions, as communication is important for cooperation [9] and user involvement is important for IT effectiveness [22]; (f) taking initiative to learn IT knowledge so that he/she can communicate with the IT personnel well; (g) proposing suggestions to IT department so that IT may better meet the business needs, and (h) taking initiative to communicate with IT personnel, as past research shows that IT knowledge, elear goals and objectives, and the alignment between IT and business objectives are important to achieve IT effectiveness [9, 23-26].

The above initiatives are discretionary and difficult to be enforced as in-role requirements. Employees may decide not to cooperate and not to be engaged in these behaviors. If this is the case, even though the frequency of use, the time of use or the number of accesses are the same, IT effectiveness may be different.

Based on the above discussion, we cmay see that both in-role IT use behaviors and discretionary IT cooperation behaviors will contribute to IT effectiveness. However, in the long run, discretionary IT cooperation behaviors should be critical for achieving IT effectiveness, as what they coneern is the CT effectiveness and not the IT usage. The traditional measurements of EJ use may not have captured these discretionary behaviors in IT use. Therefore, it is necessany to further analyze the role of these discretionary IT use behaviors for IT effectiveness.

The above discussion focuses on OCB in IT use. However, according to the results in organizational behavior research (7), there are also CWB in IT use. We call CWB in IT use as discretionary IT resistance bethavior. Based on the IT literature and our interviews with CIOs, a set of discretionary IT resistance behaviors are generated as below: (a) discussing with other employees about the useless of IT, (b) complaining frequently to other employees that IT design is not user friendry, (c) refusing to learn new IT knowledge and insisting on using the old method to carry out work duties, (d) finding excuses to be absent for IT skill-training program, (e) dêmanding other employees or departments to change their work methods to accommodate their avoidance of IT use. These behaviors are detrimental to IT effectiveness, as past research has shown that user resistance to change is a bottleneck for IT implementation [23].

As a summary, the in-role behaviors and discretionary behaviors in IT use discussed above are shown in Table 1. The list of behaviors can be further developed into measurement items of OCB and CWB in IT context. 
Table 1. In-role Behaviors and Discretionary Behaviors in IT Use

\begin{tabular}{|c|c|c|}
\hline & Constructs & Behavior description \\
\hline In-role & In-role IT use behavior & $\begin{array}{l}\text { (a) participating the training activities organized by the company } \\
\text { according to the requirements; } \\
\text { (b) inputting data to IT systems according to the requirements; } \\
\text { (c) outputting data from IT systems according to the requirements. }\end{array}$ \\
\hline$O C B$ & $\begin{array}{c}\text { Discretionary IT cooperation } \\
\text { behavior }\end{array}$ & $\begin{array}{l}\text { (a) taking initiative to learn the skill about IT use to make sure they } \\
\text { can operate IT well; } \\
\text { (b) reporting the operation problems they met immediately to make } \\
\text { sure IT work well; } \\
\text { (c) providing suggestions to make data input and output møre } \\
\text { conveniently and more friendly; } \\
\text { (d) eagerly helping other people to solve IT problems; } \\
\text { (e) taking initiative to discuss with colleagues about IT solutions; } \\
\text { (f) taking initiative to learn IT knowledge so that he/she can } \\
\text { communicate with the IT personnel well; } \\
\text { (g) proposing suggestions to dT department so that IT may better } \\
\text { meet the business needs; } \\
\text { (h) taking initiative to communicate with IT personnel. }\end{array}$ \\
\hline$C W B$ & IT resistance behavior & $\begin{array}{l}\text { (a) discussing with ther employees about the useless of IT; } \\
\text { (b) complaining frequently to other employees that IT design is not } \\
\text { user friendly; } \\
\text { (c) refusing to fearn new IT/knowledge and insisting on using the old } \\
\text { method to carry out work duties; } \\
\text { (d) finding excuses to be absent for IT skill-training program; } \\
\text { (e) demanding other employees or departments to change their work } \\
\text { methods to accommodate their avoidance of IT use. }\end{array}$ \\
\hline
\end{tabular}

\subsection{Emotion and discretionary behavior}

Research in organizational behavion field has shown that emotion has important effect on individual behavior. Spector and Fox (2002) emotion-centered model shows that emotion plays a critical role in discretionaty behavior. Negative emotions will tend to increase the likelihood of CWB and positive emotions increase the likelihood of OCB. Emotion motivates discretionary behavior which can reduce negative feelings and enhance positive feelings, and which may be benefit or harmful to an individual or an organization. In most cases, an individual who feels bae will engage in retaliation in order to make himself or herself feel better. Events which induce positive emotion will encourage an individual to become more involved in work and enhance his or her pleasurable feelings [15]. Lee and Alen (2002) showed that discretionary behaviors were predicted by both emotions and cognitions[14]. However, past research has focused on the cognition antecedents of IT use, and paid little attention to the discretionary behaviors in IT use and their emotion antecedents[27].

Based on the above discussion, we believe when employees have positive emotions towards the application of IT such as joy, relief, hope and cheer, they will tend to engage in discretionary IT cooperation behaviors, which will contribute positively to IT effectiveness. When employees have negative emotions towards the application of IT such as anger, frustration, sad and fear, they will tend to engage in discretionary IT resistance behaviors, which will contribute negatively to IT effectiveness. Therefore, we suggest the following propositions.

Proposition 1: Positive emotion will have positive effect on IT effectiveness through discretionary IT cooperation behavior.

Proposition 2: Negative emotion will have negative effect on IT effectiveness through discretionary IT resistance behavior. 
However, individual characteristic and organizational culture may moderate the relationship between emotion and discretionary behavior. One important individual characteristic stated in emotion management literature is emotional intelligence, which refers to the ability to handle issue and problem involving emotion[28, 29]. Employees with high emotional intelligence are able to understand and control their emotion more effectively. Furthermore, they are able to use emotion as a driver for the job performance[30]. Therefore, in IT context, we believe emotional intelligence will enhance the impact of positive emotion on OCB in IT use and reduce the impact of emotion on CWB in IT use. Thus, the following proposition is suggested.

Proposition 3: Employees' emotional intelligence will moderate the relationship between emotion and discretionary IT usage behavior. It will enhance the relationship between positive emotion and discretionary IT cooperation behavior, and reduce the perationship between negative emotion and discretionary IT resistance behavior.

Additionally, research in organizational behavior field has shoy that the style of organizational coping with employee emotion will affect organization ability to prevent negative outcome of employee emotion [31]. Therefore we believe of ganizational culture may play an important role in the relationship between emotion and discretionary IT usage behavior. If organizational culture encourages the employees to help and support each other, the effect of positive emotion on IT cooperation behavior will be enhanced while the detrimental effect of negative emotion on IT resistance behavior will be reduced. Therefore, the following proposition is suggested.

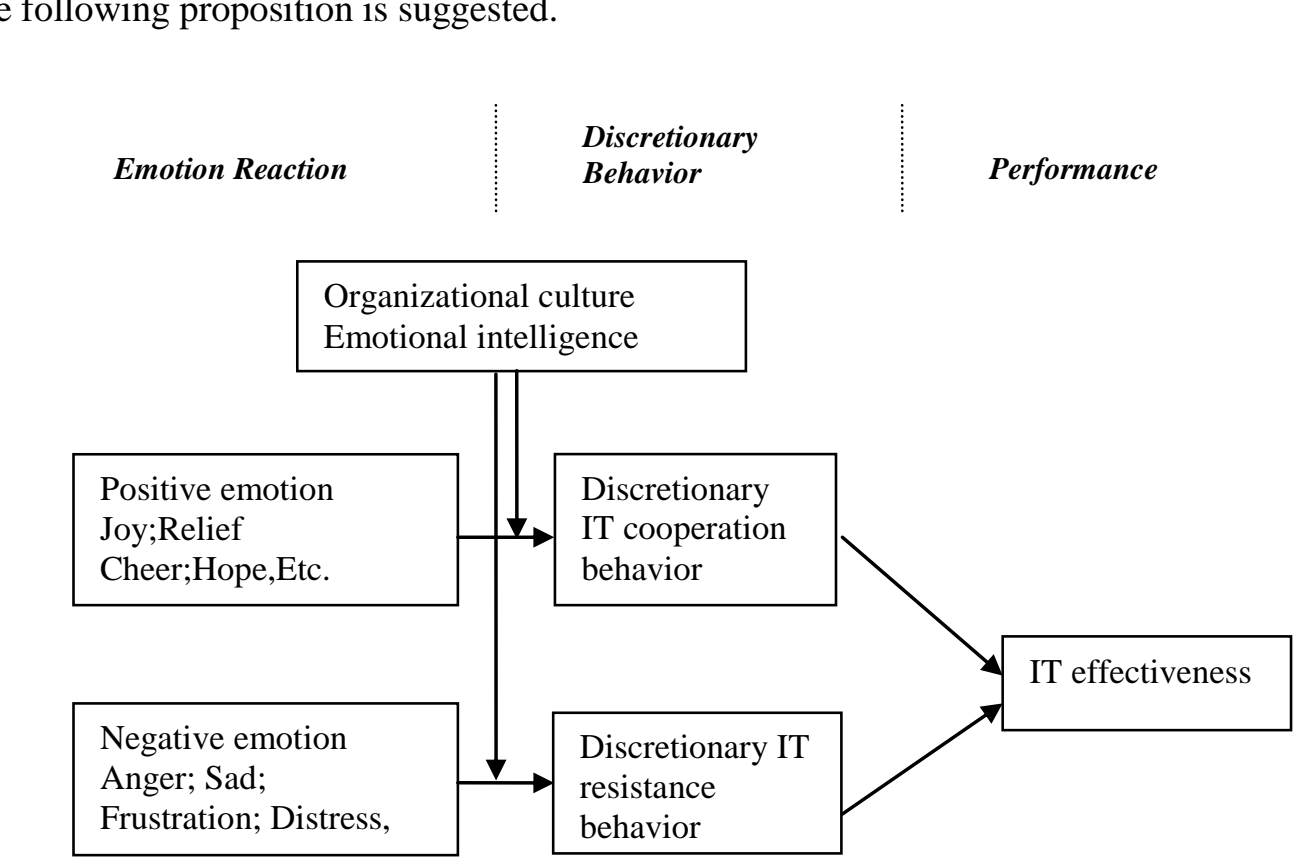

Figure 1. A process model towards IT effectiveness

Proposition 4: Organizational culture which encourages employees to help and support each other will moderate the relationship between emotion and discretionary IT usage behavior. Such culture will enhance the relationship between positive emotion and discretionary IT cooperation behavior, and reduce the relationship between negative emotion and discretionary IT resistance behavior.

Based on the above discussion, a new framework which links emotion factors and IT effectiveness is showed in Figure 1. 


\section{Discussion}

IT usage may not necessarily lead to positive individual and organizational impact. With less clear in-role behaviors, discretionary behaviors become more important for achieving job performance. The application of IT needs cooperation and interaction among employees. This means that discretionary behaviors are critical to materialize IT impact. Traditional measurements of IT use cannot fully capture all the discretionary IT use behaviors which eventually will contribute to IT effectiveness. Most research of IT use antecedents focuses on the cognition factors, while the emotion antecedent research is still limited[27]. This paper attempts to conduct a pilot analysis about the discretionary behavior in IT context, and try to explain its emotion antecedents and its outcome. We propose a new process model to explain how emotion factors influence IT effectiveness through the discretionary behaviors.

Based on the research in organizational behavior, we divide the IT use beharior into inrole behavior and discretionary behavior, and make a preliminary recognition of the discretionary behavior descriptions in IT context. Specially we recognize the OCB in IT context called discretionary cooperation behavior and the CWB in IT context called discretionary resistance behavior. Based on the organzational behavioy theory, we propose that positive emotion will motivate the discretionary IT cooperation behavior while negative emotion will motivate the discretionary IT resistance behavior. However, the extent to which emotion motivates discretionary IT cooperation behaviofor discretionary IT resistance behavior is affected by individual emotion intelligence and organizational culture. The discretionary IT cooperation behavior and discretionary IT resistance behavior will then lead to different IT effectiveness.

There are several important theoretical and practical implications from articulating a process model towards IT effectiveness. First it helps us understand why IT use does not always lead to IT effectiveness, and thus helps us move the research focus to the nature of IT use and further analyze the diseretionary IT u'se behavior. Second, it provides a framework for further empirical testing the conditions and processes which are associated with IT effectiveness. Particalarly, it revea s the areas which need further research. As stated above, a lot of IT research has been done on the cognitive antecedents of IT use. By careful extension of the organizational behavio literature, we learn more about the nature of IT use behavior, and show what kinds of IT use behaviors will affect IT effectiveness in the long run and that emotions may play important roles in such behaviors. The process connecting the emotions to the IT effectiveness reveals that a lot of further research needs to be done on what constitutes the OCB in IT use and the CWB in IT use, how emotions affect discretionary cooperation behaviors and discretionary resistance behaviors, how management can intervene employee emotions to better outcomes. Third, further empirical work based on this framework may provide ractical guidance in several important IT management areas such as how to better manage the discretionary IT usage behaviors in organization and how to better manage employee emotions involved the application of IT.

As stated in our earlier discussion, we believe it is important to conduct empirical test about the relationship between emotion and discretionary IT usage behavior, and further empirically examine the relationship between discretionary IT usage behavior and IT effectiveness, as less is known about them. We have conducted a pilot interview with the CIOs in 3 companies and reviewed the IT literature to collect the discretionary behavior descriptions which may be further developed into measurement items of OCB and CWB in IT studies. In the next step, we believe case studies and panel interview should be conducted to further collect the OCB and CWB descriptions in IT context. Then large sample survey should be conducted to collect data to choose and examine the measurement items about the constructs and empirically test hypotheses about the relationships and the moderators between 
discrete emotions and the discretionary IT usage behaviors, and the relationships between discretionary IT usage behaviors and IT effectiveness.

In summary, we propose a conceptual process model which not only provides an explanation of how emotion affects IT effectiveness and why simply IT use may not always lead to IT effectiveness, but also reveals the areas for further research to better understand IT effectiveness and IT use such as developing the measurement items for discretionary cooperation behavior and discretionary resistance behavior, analyzing the emotion antecedents of such discretionary IT usage behaviors and the moderators between emotions and discretionary IT usage behaviors.

\section{Acknowledgements}

This research was supported by the National Natural Science Foundation of China (No. 71202098; No. 71072091), Natural Science Foundation of Guangdong Province (No. S2011040001064), and a research grant from Guangdong University Jof Finance and Economics (No. 10ZD63001).

\section{References}

[1] W. H. Delone and E. R. McLean, "The DeLore and McLean/Model of Information Systems Success: A Ten-Year Update", Journal of Management Information-Systems, vol.19, no. 4, (2003), pp. 9-30.

[2] F. D. Davis, "Perceived Usefulness, Perceived Ease of Use, and User Acceptance of Information Technology", MIS Ouarterly, vol 13,no. 3, (1989), pp. 3,19-340.

[3] A. Beaudry and A. Pinsonneault, "The Other Side of Acceptance: Studying the Direct and Indirect Effects of Emotions on Information Technology Use", MIS Quarterly, vol.34, no. 4, (2010), pp. 689710.

[4] V. Venkatesh, M. G. Mofris, G. B. Davis and F. D. Davis, "User Acceptance of Information Technology: Toward a Nified View", MIS Quarterly, vol. 27, no. 3, (2003), pp. 425-478.

[5] W. H. Delone and E. R Maclean, "Information System Success: The Quest for the Dependent Variable", Information Systems Résedrch, vol. 3, no. 1, (1992), pp.60-95.

[6] M. Rotundo and P. R. S.ckett, "The Relative Importance of Task, Citizenship, and Counterproductiye Performance to Global Ratings of Job Performance: A Policy-Capturing Approach", Journal of Applied) Psychology, vol. 87, no. 1, (2002), pp. 66-80.

[7] E. H. O. Boyle, "A Test of the General CWB-OCB Emotion Model”, Doctoral Dissertation, Virginia Commonwealth Univepsity (2010).

[8] J. R. Knapp, T. Dalziel and M. W. Lewis, "Governing Top Managers: Board Control, Social Categorization, and Their Unintended Influence on Discretionary Behaviors", Corporate Governance; An International Review, vol. 19, no. 4, (2011), pp. 295-310.

[9] H. Akkermans, and K. V. Helden, "Vicious and Virtuous Cycles in ERP Implementation: A Case Study of Interrelations between Critical Success Factors", European Journal of Information Systems, vol. 11, no. 1, (2002), pp.35-46.

[10] F. D. Davis, R. P. Bagozzi, and P. R. Warshaw, "Extrinsic and Intrinsic Motivation to Use Computers in the Workplace", Journal of Applied Social Psychology, vol. 22, no. 14, (1992), pp.1111-1132.

[11] M. Fishbein and I. Ajzen, "Belief, Attitude, Intention and Behavior: An Introduction to Theory and Research", Addison-Wesley Reading, MA (1975).

[12] S. Taylor and P. Todd, “Assessing IT Usage: The Role of Prior Experience”, MIS Quarterly, vol.19, no. 4, (1995), pp. 561-570.

[13] J. N. Choi, S. Y. Sung, K. Lee, and D.-S. Cho, "Balancing Cognition and Emotion: Innovation Implementation as a Function of Cognitive Appraisal and Emotional Reactions toward Innovation", Journal of Organizational Behavior, vol. 32, no. (2011), pp. 107-124.

[14] K. Lee and N. J. Allen, "Organizational Citizenship Behavior and Workplace Deviance: The Role of Affect and Cognitions", Journal of Applied Psychology, vol. 87, no. 1, (2002), pp.131-142.

[15] P. E. Spector and S. Fox, "An Emotion-centered Model of Voluntary Work Behavior: Some Parallels between Counterproductive Work Behavior and Organizational Citizenship Behavior", Human Resource Management Review, vol. 12, no. (2002), pp. 269-292. 
[16] W. W. Chin and A. Gopal, "Adoption Intention in GSS: Relative Importance of Beliefs, "The Data Base for Advances in Information Systems, vol. 26, no. 2/3, (1995), pp.42-64.

[17] A. Bhattacherjee, "Understanding Information Systems Continuance: An Expectation-Confirmation Model", MIS Quarterly, vol. 25, no. 3, (2001), pp. 351-370.

[18] R. T. Cenfetelli, "Getting in Touch with Our Feelings towards Technology", Academy of Management Proceedings, (2004) Augut 6-11, New Orleans.

[19] S. A. Brown, R. M. Fuller, and C. Vician, "Who's Afraid of the Virtual World? Anxiety and Computer Mediated Communication", Journal of the Association for Information Systems, vol. 5, no. 2, (2004), pp.79-107.

[20] S. Han, J. S. Lerner and D. Keltner, "Feelings and Consumer Decision Making: The AppraisalTendency Framework, Journal of Consumer Psychology, vol. 17, no. 3, (2007), pp.158-168.

[21] R. Heckman and A. Guskey, "The Relationship between Alumni and University: Toward $x$ Theory of Discretionary Collaborative Behavior”, Journal of Marketing, vol. no. 1, (1998), pp. 97-112

[22] M. A. Harris and H. R. Weistroffer, "A New Look at the Relationship between User Involvement in Systems Development and System Success", Communications of the Association fo Information Systems, vol. 24, no. 6, (2009), pp. 739-756.

[23] M. J. Gallivan, "Organizational Adoption and Assimilation of Cómplex Teênological Innovations: Development and Application of a New Framework", The Data Base for Advances in Information Systems, vol. 32, no. 3, (2001), pp. $51-85$.

[24] H.-F. Lin, "Empirically Testing Innovation Characteristic ard Organizational Learning Capabilities in E-Business Implementation Success", Internet Research, vol. 18, no 1. (2008), pp. 60-78.

[25] M. Huang and K. Xie, "First-line and Middle Mapager IT Application Competence Model", Chinese Journal of Management, vol. 9, no. 5, (2012), pp. 692-698.

[26] A. S. Ortiz, "Testing a Model of the Relationships among Organzational Performance, IT-business Alignment, and IT Governance, Doctoral Dissertation”, University of North Texas (2003).

[27] M. Huang, "Another Perspective of IS Success: AHow Emotion Affecting IS Effectiveness?", Proceedings of the 2013 International Conference on Information Technology and Electronic Commerce,(2013) Dec 28-29, Harbin, China.

[28] K. S. Law, C.-S. Wong and L. J. Song, "The Construct and Criterion Validity of Emotional Intelligence and Its Potential Utility for Management Studies", Journal of Applied Psychology, vol. 89, no. 3, (2004), pp. 483-496.)

[29] C.-S. Wong and $K_{\odot}$ S A Aw, "The Effects of Leader and Follower Emotional Intelligence on Performance and Attitude An Exploratory Study", The Leadership Quarterly, vol. 13, (2002), pp. 243-274.

[30] D. L. Joseph and D. A. Newman, Emotional Intelligence: An Integrative Meta-Analysis and Cascading Mode)", Journal of Applied Psychology, vol. 95, no. 1, (2010), pp.54-78.

[31] N. M. Ashkanasy and G. S. Daus, "Emotion in the Workplace: The new challenge for managers", Academy of Management Executive, vol. 16, no. 1, (2002), pp.76-86.

\section{Authors}

Manhui Húang received her Ph.D. in management information system in 2009 from Business School, Sun Yat-sen University, China. She is currently an associate professor at Information Science School, Guangdong University of Finance and Economics. Her research foctises on IT management, IT adoption, and human behavior analysis in IT context.

Jinghua Xiao received her Ph.D. in management information system in 2007 from Business School, Sun Yat-sen University, China. She is currently an associate professor at Business School, Sun Yat-sen University. Her research focuses on IT management and interorganizational IT governance.

Kang Xie received his Ph.D. in management information system in 1999 from Renmin University of China. He is currently a professor at School of Business, Sun Yat-sen University. His research focuses on IT management and inter-organizational IT governance. 
International Journal of Multimedia and Ubiquitous Engineering Vol.9, No.5 (2014)

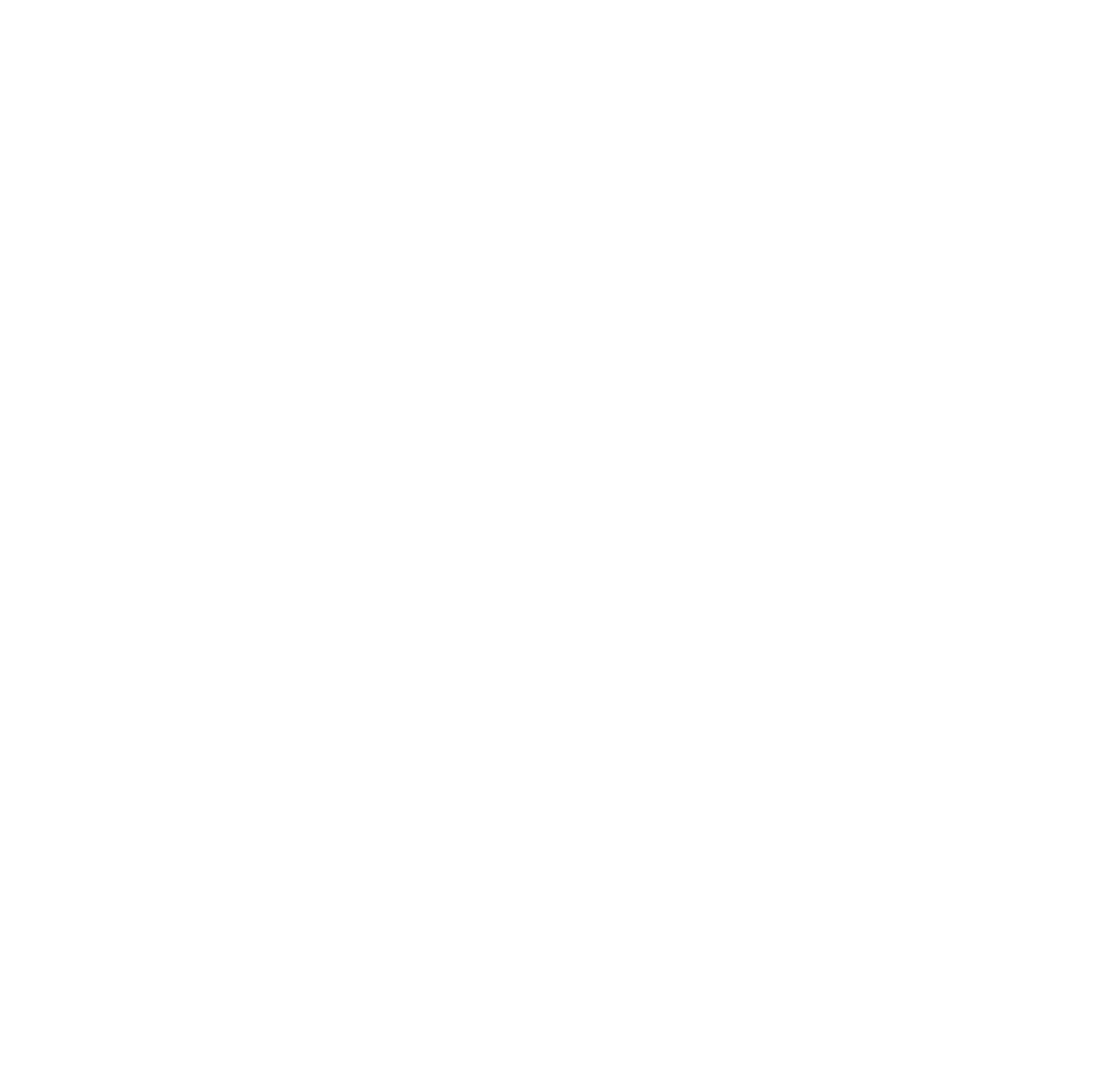

